\title{
Towards Embedded System for Real-time Feedback in Table Tennis Based on 2.4 GHz Wireless Sensor
}

\author{
Rabee M. Hagem \\ School of Engineering \\ Centre for Wireless Monitoring and \\ Applications/Brisbane/Queensland/Australia \\ Collage of Engineering/ Computer \\ Engineering Department/ \\ Mosul University/Erbil//raq
}

\begin{abstract}
Real time monitoring and feedback are important issues for athletes and coaches in order to access the optimum performance. Table tennis strokes for different phases including (forehand and backhand drive, forehand and backhand push, forehand and backhand topspin) are important to be monitored and calculated in real time. nCore 2.0 wearable wireless sensor that is designed in the center for wireless monitoring and applications at Griffith university is used with some hardware and software modifications. This sensor is used after adding the $2.4 \mathrm{GHz}$ module in order to send real time data. The sensor is connected on an athlete wrist and the RF signal strength is measured using the RF explorer (RF handheld spectrum analyzer) in order to design embedded system for real time monitoring and feedback. Data is wirelessly transmitted to a tablet on the coach's hand in real time. The flexible sensor allows for recording, analyzing kinematics information on the racket motion and finally sending it in real time to a coach. Experiments are conducted in order to monitor the $2.4 \mathrm{GHz}$ signal strength. The results showed that this system is feasible to build a reference motion database which is very useful for athletes and coaches.
\end{abstract}

\section{General Terms}

Digital Systems, Human-Computer Interaction, Embedded Systems.

\section{Keywords}

Embedded Systems, Table Tennis, Wearable Wireless Sensor, Real time Monitoring and Feedback, Wireless Link Reliability.

\section{INTRODUCTION}

Human movement during sporting activates plays an important role for coaches to assess athlete's performance and reach the optimum goal. The development of micro electronical (MEMS) based inertial sensors with low cost, low power and small size allows the researchers to do so. The main focus in this paper is on using sensors in sports. However, the sensor technology can be deployed in different areas including health and human movement [1-3]. To measure and analyze player's movements three dimensional inertial sensors based on accelerometers are used by connect them on the athlete wrist's to record the data and send it in real time. Wireless inertial measurement unit (WIMU) called nCore 2.0 is used. This unit is development and implemented at Griffith University/ center for wireless monitoring and applications (CWMA). However, modifications were applied on the hardware and software in order to use it for the purpose of this paper. The value that the accelerometer measure contains two components: "static" acceleration that depends on the orientation of the sensors to the gravity. The second acceleration component is "dynamic" which is corresponding to 2 nd derivative of the position relative to a given axis [4]. The nCore 2.0 wireless sensor is programmed using $\mathrm{C}$ language with Atmel Studio 6.1 in order to generate the 2.4 $\mathrm{GHz}$ RF signal. This signal is transmitted wirelessly using the IEEE protocol ( $2.4 \mathrm{GHz}$ band). A rechargeable battery is used to power the unit. The main goal of this paper is to measure the $2.4 \mathrm{GHz} \mathrm{RF}$ signal from a wearable wireless sensor connected on an athlete wrist's during playing table tennis. The measurements are done in different positions for the player in order to design later an embedded system for this game for real time feedback between athletes and their coaches. This paper is organized as follows: In section 2 the system design is presented discussing the nCore 2.0 wearable wireless sensor design and the added RF module. In section 3 the experiments and the results are described based on the RF signal strength experiment. Finally in section 4 conclusions are drawn and discussion of the directions for the future work are presented.

\section{SYSTEM DESIGN}

In this paper the nCore2.0 wireless sensor is used. The core of the sensor is the microcontroller unit (ATmega 324P) which is a very popular processor to be used for embedded applications all over the world. This Atmel microcontroller features 8 channels for analogue input which allow connect different sensors. It contains also 1 kbyte of EEPROM with a 32 kbytes flah memory for programming and $32 \mathrm{I} / \mathrm{O}$ lines. The communication with peripheral devices can be done through SPI. This microcontroller can be operating at variable clock rates from $(\mathrm{KHz}$ to $\mathrm{MHz})$ with 10 MIPS processing throughput. The sensor includes on board 2 Mbytes memory (AT45DB161P) for data storage. The tri-axial accelerometer (MMA7260QT) with a programmable gain with up to $\pm 6 \mathrm{G}$ range is included on the sensor. The easy communications with a PC can be done through the on-board USB chip provided (CP2102). This sensor is easy customizable for specific applications by including additional external analogue and digital connection sockets. $3.6 \mathrm{~V}$ battery with a maximum capacity of $240 \mathrm{~mA}$ is used to power the sensor. This sensor is designed at Griffith university with dimensions of $52 \mathrm{~mm} \times 34 \mathrm{~mm} \times 12 \mathrm{~mm}(\mathrm{~L} \times \mathrm{W} \times \mathrm{H})$ with $22 \mathrm{~g}$ weight [5]. This sensor is used after adding hardware and software modifications. The addition hardware for this sensor is the RF module (NRF24L01+) with a built in PCB monopole antenna as shown in figure 1 . Figure 2 shows the block diagram of this sensor [6-8]. 


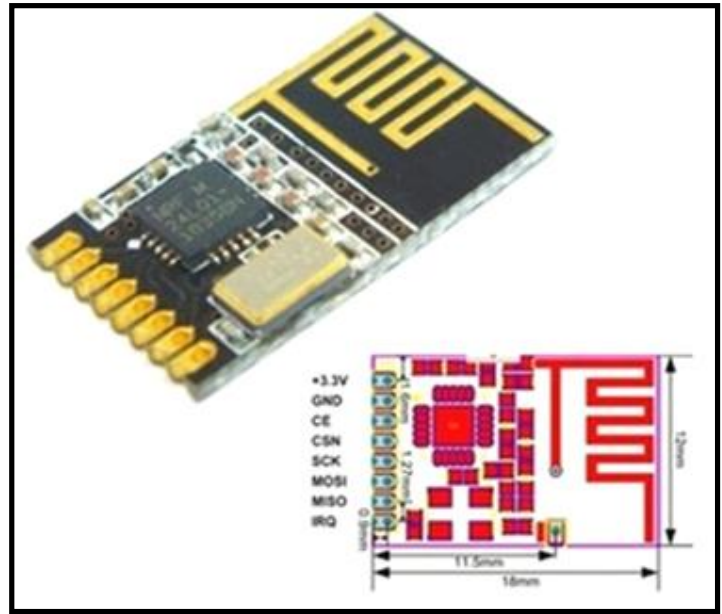

Fig 1: The RF module that is added to the nCore $\mathbf{2 . 0}$ sensor.

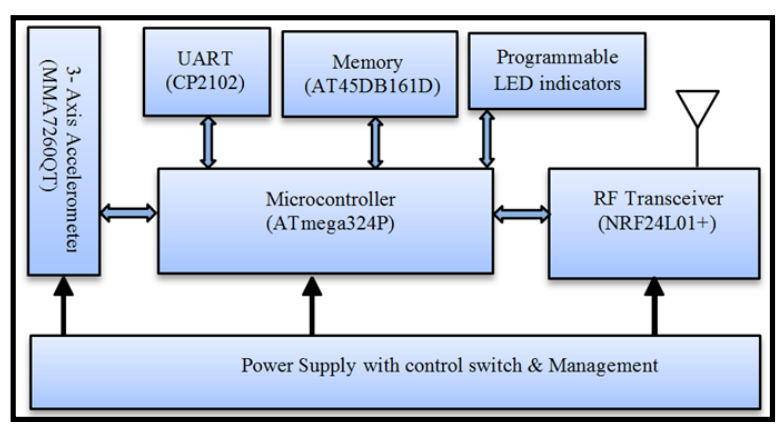

Fig 2: Block diagram of the nCore2.0 wireless sensor after adding the RF Module [5-8].

Figure $3 \mathrm{a}$ and $3 \mathrm{~b}$ shows the real nCore 2.0 wireless sensor before and after adding the RF module on it respectively.

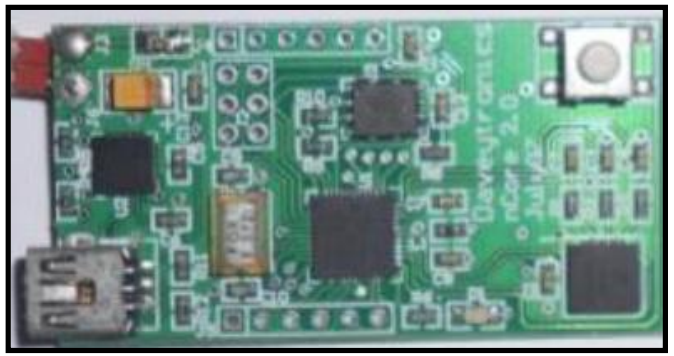

Fig 3a: nCore 2.0 before adding the RF module [6].

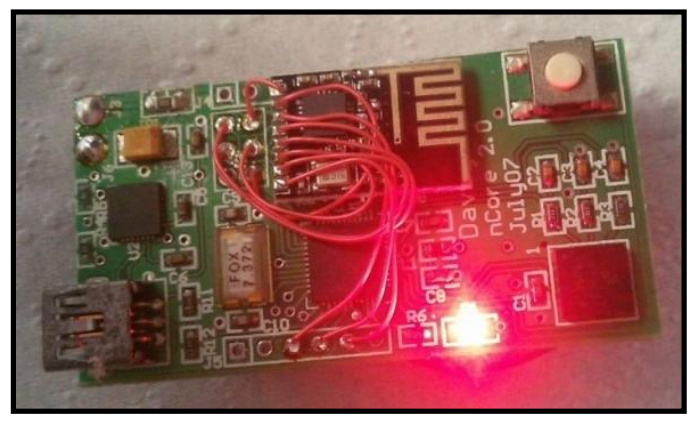

Fig 3b: nCore 2.0 after connecting the RF module.

\section{EXPERIMENTS AND RESULTS}

Experiments are conducted in this paper in order to check the system feasibility. The experiment is by checking the RF (Radio Frequency) signal strength of the $2.4 \mathrm{GHz}$ using RF explorer device (affordable and portable device with USB data interface) while the athlete in the field playing table tennis. The RF module on the nCore 2.0 is programmed to send signal continuously at $2.4 \mathrm{GHz}$ and the LED is flashing as indication of sending signal. In this experiment, right handed professional athlete is asked to play for around five minutes and the RF explorer is used to record the signal strength every five seconds. The coach with the tablet and the RF explorer was two meter away from the table tennis. Snigat 9 software was used to capture the RF signal by connecting the RF explorer device through the USB to the coach tablet and record the signal strength every 5 seconds (using timer) in order to analyze it later. The RF module that we added to the nCore 2.0 was (NRF24L01+). This module is based on Nordic nRF24L01, high integrated, ultra-low power (ULP) 2Mbps $\mathrm{RF}$ transceiver for the $2.4 \mathrm{GHz}$ ISM (Industrial, Scientific and Medical) band. The transmit power is $+7 \mathrm{dBm}$ and the receiver sensitivity is $-90 \mathrm{dBm}$ [9]. Figure 4 shows the nCore 2.0 with RF explore device.

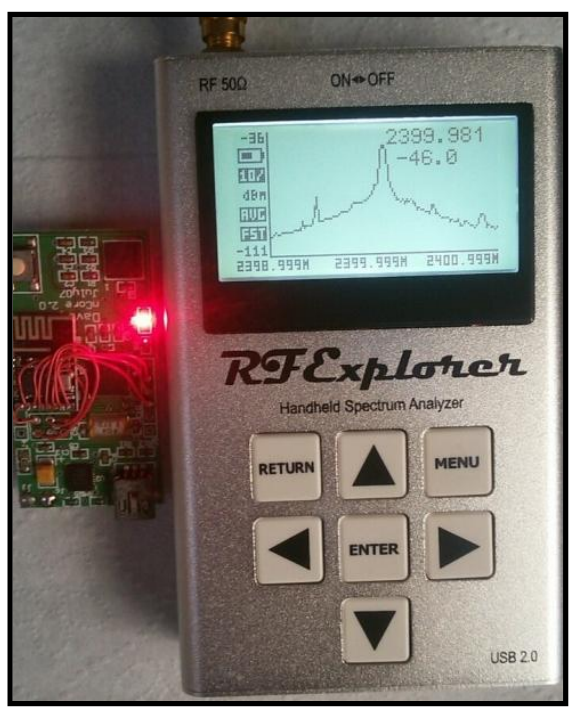

Fig 4: The nCore 2.0 with the RF explore device.

Figure 5 shows a sample from the RF explore screen captured on the tablet screen which represent the signal strength in $\mathrm{dBm}$ with different frequencies. The $2.4 \mathrm{GHz}$ signal can be noticed as a peak.

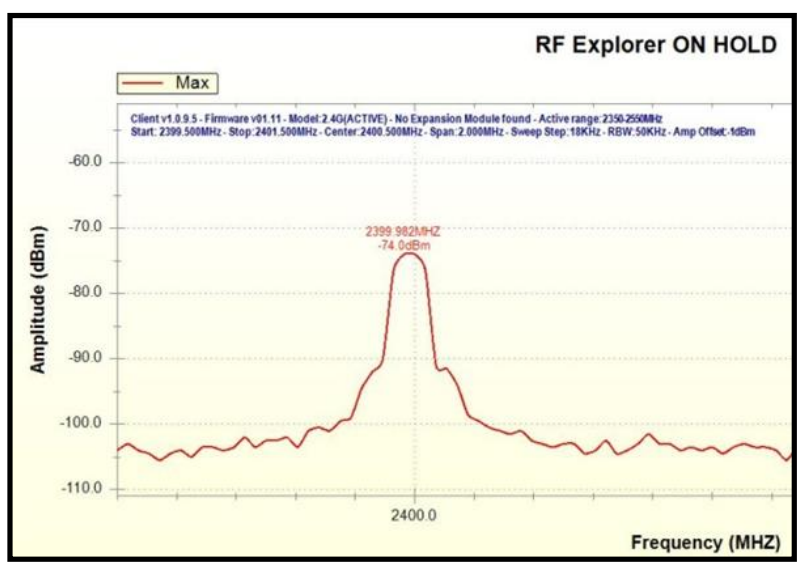

Fig 5: signal strength with frequency.

The data is recorded easily after finishing the experiment because it is captured using the snigat 9 software that can capture video, image and sound. Matlab is used to plot the data which represent the signal strength in $\mathrm{dBm}$ with time for 
different positions of a table tennis player. Fig. 6 shows the received signal in $\mathrm{dBm}$ with time and compared it with the receiver sensitivity which represents the minimum signal power required for the communication to be successful. The link margin represents the received power subtracting the receiver sensitivity. If this value is greater than zero this means that the communication link is successful otherwise if it is negative this means that the commination system cannot achieve the required rang. The figure showed that the received signal is greater than the receiver sensitivity with different positions and different strokes of the athlete during playing the table tennis. This means that the communication link is successful and the data should be received correctly.

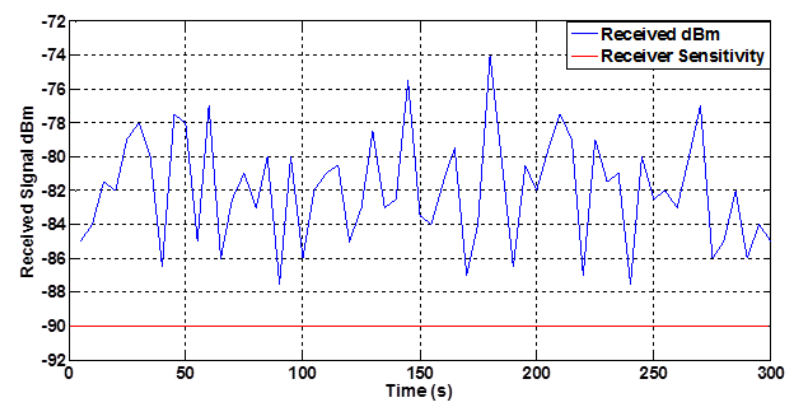

Fig 6: Received RF signal in $\mathrm{dBm}$ with time. The red line represents the receiver sensitivity.

\section{CONCLUSION AND FUTURE WORK}

This paper presents a system based on nCore 2.0 wireless wearable sensor designed and implemented at Griffith University for monitoring the RF signal strength in table tennis. Hardware and software modifications on this sensor are made in order to use it in this paper. This is the first step towards building embedded system in table tennis that will be including monitoring and real time feedback for athletes and coaches in order to reach the optimum performance. The results including the received signal in $\mathrm{dBm}$ with time and for different positions for the athlete during the table tennis game showed that this system is feasible. For future work two nCore 2.0 wireless sensors with RF modules will be used one as transmitter and another one as a receiver to send real time data and check the packet error rate in order to send real time feedback between the coaches and the athletes.

\section{ACKNOWLEDGMENTS}

The author would like to thank Prof. David Victor Thiel, Professor of Griffith University/school of Engineering for providing the nCore 2.0 wireless sensors. Thanks for the participants and helpers to performer the experiments. Ethics approval was granted for the human studies in this research (ENG/05/10/HREC).

\section{REFERENCES}

[1] J. L. a. D. A. J. Hugo G. Espinosa, "The Inertial Sensor: A Base Platform For Wider Adoption In Sports Science Applications," Journal of Fitness Research, vol. 4, pp. 13-20, April 2015.

[2] D. A. James, D. E. Thiel, K. J. Allen, B. Abell, S. Kilbreath, G. M. Davis, et al., "Technology and health: Physical activity monitoring in the free living environment," Procedia Engineering, vol. 34, pp. 367372, 2012.

[3] D. V. Thiel, H. G. Espinosa, G. M. Davis, E. Dylke, N Foroughi, and S. L. Kilbreath, "Arm Movement: The Effect of Obesity on Active Lifestyles," Procedia Engineering, vol. 60, pp. 182-187, 2013.

[4] F. B. Eric Boyer, François Phal and Sylvain Hanneton, "Low-cost motion sensing of table tennis players for real time feedback,", The $13^{\text {th }}$ ITTF Sports Science Congress, Paris, France, May 11-12, 2013.

[5] N. Davey, A. Wixted, Y. Ohgi, and D. James, "A low cost self contained platform for human motion analysis," The impact of technology on sport II, pp. 101-111, 2008.

[6] R. M. Hagem, "Real Time Evaluation of Swimmers Performance Based on an Optical Wireless Communication System," Ph.D Thesis, Griffith University, 2013.

[7] R. M. Hagem, S. G. O. Keefe, T. Fickenscher, and D. V. Thiel, "Self Contained Adaptable Optical Wireless Communications System for Stroke Rate During Swimming," IEEE Sensors Journal, vol. 13, pp. 31443151,2013

[8] H. A. Sabti and D. V. Thiel, "Node Position Effect on Link Reliability for Body Centric Wireless Network Running Applications," IEEE Sensors Journal, vol. 14, pp. 2687-2691, 2014

[9] N. Semiconductor. (2008, 2013). nRF24L01+ SingleChip $2.4 \mathrm{GHz}$ Transceiver Preliminary Product Specification V1.0. Available:http://www.nordicsemi.com/eng/Products/2.4 GHz-RF/nRF24L01 